\title{
Stem cells conditioned medium: a new approach to skin wound healing management
}

Type: Article

Abstract:

Stem cell biology has gained remarkable interest in recent years, driven by the hope of finding cures for numerous diseases including skin wound healing through transplantation medicine. Initially upon transplantation, these cells home to and differentiate within the injured tissue into specialised cells. Contrariwise, it now appears that only a small percentage of transplanted cells integrate and survive in host tissues. Thus, the foremost mechanism by which stem cells participate in tissue repair seems to be related to their trophic factors. Indeed, stem cells provide the microenvironment with a wide range of growth factors, cytokines and chemokines, which can broadly defined as the stem cells secretome. In in vitro condition, these molecules can be traced from the conditioned medium or spent media harvested from cultured cells. Conditioned medium now serves as a new treatment modality in regenerative medicine and has shown a successful outcome in some diseases. With the emergence of this approach, we described the possibility of using stem cells conditioned medium as a novel and promising alternative to skin wound healing treatment. Numerous pre-clinical data have shown the possibility and efficacy of this treatment. Despite this, significant challenges need to be addressed before translating this technology to the bedside.

\begin{tabular}{|c|l|}
\hline Author & Jayaraman, P. ;Nathan, P. ;Vasanthan, P. ;Musa, S. ;Govindasamy, V. \\
\hline Source & Cell Biology International \\
\hline ISSN & $1065-6995$ \\
\hline DOI & $10.1002 /$ cbin.10138 \\
\hline Volume (Issue) & $37(10)$ \\
\hline Page & $1122-1128$ \\
\hline Year & 2013 \\
\hline
\end{tabular}

Keyword:

cytokines;growth factors;host tissues;paracrine activities; regenerative medicine;tissue repair;paracrine mechanisms;stromal cells;in-vitro;cancer;Cell Biology

URL:

- http:/lapps.webofknowledge.com search via Accession No >> 000323932000010

- Full text available at : http://onlinelibrary.wiley.com/doi/10.1002/cbin.10138/pdf 